\title{
Prednisone for the Prevention of Paradoxical Tuberculosis-Associated IRIS
}

\author{
G. Meintjes, C. Stek, L. Blumenthal, F. Thienemann, C. Schutz, J. Buyze, \\ R. Ravinetto, H. van Loen, A. Nair, A. Jackson, R. Colebunders, G. Maartens, \\ R.J. Wilkinson, and L. Lynen, for the PredART Trial Team
}

ABSTRACT

BACKGROUND

Early initiation of antiretroviral therapy (ART) in human immunodeficiency virus (HIV)-infected patients who have tuberculosis reduces mortality among patients with low CD4 counts, but it increases the risk of paradoxical tuberculosis-associated immune reconstitution inflammatory syndrome (IRIS).

\section{METHODS}

We conducted this randomized, double-blind, placebo-controlled trial to assess whether prophylactic prednisone can safely reduce the incidence of paradoxical tuberculosis-associated IRIS in patients at high risk for the syndrome. We enrolled HIVinfected patients who were initiating ART (and had not previously received ART), had started tuberculosis treatment within 30 days before initiating ART, and had a CD4 count of 100 cells or fewer per microliter. Patients received either prednisone (at a dose of $40 \mathrm{mg}$ per day for 14 days, then $20 \mathrm{mg}$ per day for 14 days) or placebo. The primary end point was the development of tuberculosis-associated IRIS within 12 weeks after initiating ART, as adjudicated by an independent committee.

\section{RESULTS}

Among the 240 patients who were enrolled, the median age was 36 (interquartile range, 30 to 42 ), $60 \%$ were men, and $73 \%$ had microbiologically confirmed tuberculosis; the median CD4 count was 49 cells per microliter (interquartile range, 24 to 86), and the median HIV type 1 RNA viral load was $5.5 \log _{10}$ copies per milliliter (interquartile range, 5.2 to 5.9). A total of 120 patients were assigned to each group, and 18 patients were lost to follow-up or withdrew. Tuberculosis-associated IRIS was diagnosed in 39 patients (32.5\%) in the prednisone group and in 56 (46.7\%) in the placebo group (relative risk, 0.70 ; $95 \%$ confidence interval [CI], 0.51 to $0.96 ; \mathrm{P}=0.03$ ). Openlabel glucocorticoids were prescribed to treat tuberculosis-associated IRIS in 16 patients $(13.3 \%)$ in the prednisone group and in $34(28.3 \%)$ in the placebo group (relative risk, $0.47 ; 95 \% \mathrm{CI}, 0.27$ to 0.81 ). There were five deaths in the prednisone group and four in the placebo group $(\mathrm{P}=1.00)$. Severe infections (acquired immunodeficiency syndrome-defining illnesses or invasive bacterial infections) occurred in 11 patients in the prednisone group and in 18 patients in the placebo group $(\mathrm{P}=0.23)$. One case of Kaposi's sarcoma occurred in the placebo group.

CONCLUSIONS

Prednisone treatment during the first 4 weeks after the initiation of ART for HIV infection resulted in a lower incidence of tuberculosis-associated IRIS than placebo, without evidence of an increased risk of severe infections or cancers. (Funded by the European and Developing Countries Clinical Trials Partnership and others; PredART ClinicalTrials.gov number, NCT01924286.)

The authors' full names, academic degrees, and affiliations are listed in the Appendix. Address reprint requests to Dr. Meintjes at the Institute of Infectious Disease and Molecular Medicine, Faculty of Health Sciences, University of Cape Town, Cape Town, South Africa, or at graemein@mweb.co.za.

N Engl J Med 2018;379:1915-25. DOI: $10.1056 /$ NEJMoal 800762 Copyright $\odot 2018$ Massachusetts Medical Society. 
I N RESOURCE-LIMITED COUNTRIES, A SUBstantial proportion of patients infected with the human immunodeficiency virus (HIV) present for care with advanced immunosuppression and low CD4 counts, ${ }^{1}$ and tuberculosis is the most common opportunistic infection. ${ }^{2}$ Paradoxical tuberculosis-associated immune reconstitution inflammatory syndrome (IRIS) - an immunopathologic reaction characterized by recurrent or new inflammatory features of tuberculosis that manifest shortly after the initiation of antiretroviral therapy (ART) in patients receiving antituberculosis treatment ${ }^{3}$ - occurs in $18 \%$ of patients (range, 4 to 54), according to a pooled estimate derived from 40 observational cohort studies. ${ }^{4}$ Features include recurrent symptoms, fever, lymphadenitis, and worsening pulmonary infiltrates on radiography. Low CD4 counts and a short interval between the start of antituberculosis treatment and the start of ART increase the risk of tuberculosis-associated IRIS. ${ }^{4}$ Tuberculosis-associated IRIS is associated with considerable morbidity, and $25 \%$ of patients with this syndrome are hospitalized. ${ }^{4}$

Clinical trials that evaluated the timing of ART in patients with tuberculosis showed that early initiation of ART (initiation approximately 2 weeks after the start of antituberculosis treatment) resulted in a higher survival rate than ART initiated approximately 8 weeks after the start of antituberculosis treatment in patients with CD4 counts of 50 cells or fewer per microliter. ${ }^{5}$ These findings informed World Health Organization (WHO) guidelines, which emphasize accelerating ART initiation in patients with tuberculosis and low CD4 counts. ${ }^{6}$ However, despite the survival benefit, early initiation of ART more than doubles the risk of tuberculosis-associated IRIS. ${ }^{5}$ No evidence-based strategy to prevent tuberculosisassociated IRIS exists.

Studies that evaluated adjunctive glucocorticoids for the treatment of various forms of tuberculosis showed reduced mortality among patients with tuberculous meningitis and fewer complications related to tuberculous pericarditis. ${ }^{7,8}$ Studies involving HIV-infected patients showed that the use of adjunctive glucocorticoids to treat tuberculosis was associated with adverse events, particularly Kaposi's sarcoma,, 10 but in these studies, most patients were not receiving ART when Kaposi's sarcoma was diagnosed. We previously reported the results of a placebo-controlled trial that showed that administration of prednisone resulted in a shorter duration of hospitalization and faster alleviation of symptoms than placebo when used as treatment for paradoxical tuberculosis-associated IRIS. ${ }^{11}$ We hypothesized that a low dose of glucocorticoids to attenuate the aberrant inflammatory response that underlies tuberculosis-associated IRIS during early $\mathrm{ART}^{3}$ would reduce the risk of this syndrome.

\section{METHODS}

\section{TRIAL DESIGN AND OVERSIGHT}

We conducted a phase 3, randomized, doubleblind, placebo-controlled trial to assess the efficacy and safety of prednisone for the prevention of paradoxical tuberculosis-associated IRIS in high-risk patients starting ART. The methods have been published previously ${ }^{12}$ and are provided in the protocol and statistical analysis plan, available with the full text of this article at NEJM.org.

The protocol was approved by the University of Cape Town human research ethics committee, the Institute of Tropical Medicine institutional review board, and the Antwerp University Hospital ethics committee. The trial was conducted in accordance with International Conference on Harmonisation and South African Good Clinical Practice guidelines and the principles of the Declaration of Helsinki. The authors vouch for the accuracy and completeness of the data and for the fidelity of the trial to the protocol. The University of Cape Town was responsible for the conduct of the trial and hired a consultant to monitor the trial. The funders had no role in the design of the trial; in the collection, analysis, or interpretation of the data; or in the writing of the manuscript. An independent data and safety monitoring board, which was appointed by the University of Cape Town, performed two planned reviews.

\section{TRIAL SETTING}

The trial was conducted at the Site B HIV-TB clinic in Khayelitsha, Cape Town, South Africa, a government-run outpatient primary care clinic. Khayelitsha is a township of approximately 500,000 people; in 2015, 917 cases of tuberculosis per 100,000 persons were reported in this township, and $60 \%$ of those patients were coinfected with HIV (Caldwell J, City of Cape Town Department of Health: personal communication). 


\section{PARTICIPANTS}

We enrolled patients who were 18 years of age or older, were infected with HIV, had not previously received ART and were now initiating ART, had a CD4 count of 100 cells or fewer per microliter, had microbiologically confirmed tuberculosis or a clinical diagnosis with symptomatic response to antituberculosis treatment, and had been receiving antituberculosis treatment for less than 30 days before initiating ART. The inclusion criteria were designed to result in enrollment of a high-risk group, since the incidence of tuberculosis-associated IRIS is high among patients with a CD4 count of 100 cells or fewer per microliter and among patients who start ART within 30 days after the start of antituberculosis treatment. ${ }^{4,13}$ Exclusion criteria were Kaposi's sarcoma, pregnancy, neurologic or pericardial tuberculosis, rifampin-resistant tuberculosis, hepatitis B infection, receipt of nonstandard antituberculosis treatment (the standard intensive-phase treatment was considered to be rifampin, isoniazid, pyrazinamide, and ethambutol), poor clinical response to antituberculosis treatment before the start of ART, uncontrolled diabetes, an alanine aminotransferase level of more than 200 IU per liter, an absolute neutrophil count of less than 500 per cubic millimeter, and systemic glucocorticoid use within the previous 7 days. Written informed consent was obtained for screening and again for enrollment.

\section{INTERVENTIONS AND RANDOMIZATION}

The prednisone and identical-appearing placebo tablets were manufactured by the Gulf Drug Company (Durban, South Africa) and were registered in South Africa and paid for by the trial budget. Identical packages containing prednisone or placebo were prepared at an independent offsite pharmacy and were sealed and numbered from 1 to 240. Participants were enrolled by the trial doctor, were assigned a trial number from 1 to 240 , sequentially, and received the corresponding numbered package. Randomization was performed in a 1:1 ratio with a block size of 8; the randomization sequence was prepared by an independent statistician. Patients in the prednisone group received $40 \mathrm{mg}$ of prednisone (in 5 -mg tablets) per day for 14 days, followed by $20 \mathrm{mg}$ per day for 14 days, and those in the placebo group received the equivalent number of placebo tablets. The prednisone or placebo was started within 48 hours after the initiation of ART. Group assignments remained concealed from the staff and patients throughout the course of the trial.

\section{TRIAL PROCEDURES}

Visits were scheduled at weeks 0 (when ART and the trial regimen were initiated), 1, 2, 4, 8, and 12. Patients could return to the clinic for unscheduled visits if they had new or worsening symptoms. If tuberculosis-associated IRIS symptoms persisted at week 12, follow-up was extended so that the date on which the symptoms of the syndrome resolved could be ascertained.

During the course of this trial, results of a trial of prednisolone for the treatment of tuberculous pericarditis became available; the results showed a higher incidence of cancer among HIVinfected patients in the prednisolone group than in the placebo group. ${ }^{9}$ On the basis of these results, the data and safety monitoring board for our trial advised that patients should be followed up with a visit to the clinic at 28 weeks and with a telephone call at 1 year to monitor for HIVrelated cancers. All other analyses were restricted to data obtained over 12 weeks.

When tuberculosis-associated IRIS was suspected, testing was performed (e.g., bacterial blood culturing and chest radiography) to exclude alternative causes of clinical deterioration. If tuberculosis-associated IRIS was diagnosed, the trial regimen could be stopped and open-label prednisone could be started at a dose of $1.5 \mathrm{mg}$ per kilogram of body weight per day if the episode was severe enough to warrant glucocorticoid therapy. The decision to treat with open-label glucocorticoids was made by the trial doctor, who remained unaware of the group assignment. Details regarding ART, tuberculosis treatment, and cryptococcal antigen screening, as well as reasons for stopping the trial regimen, are provided in the Supplementary Methods section in the Supplementary Appendix, available at NEJM.org.

\section{END POINTS}

The primary end point was the development of paradoxical tuberculosis-associated IRIS, determined according to the International Network for the Study of HIV-associated IRIS (INSHI) consensus case definition, within 12 weeks after initiating ART. ${ }^{14}$ All adverse events that could possibly be attributed to paradoxical tuberculosis- 
associated IRIS were adjudicated by a committee of three expert clinical investigators who were unaware of the group assignments and were not active at the trial clinic; the members of this committee decided by consensus whether INSHI criteria were met. Further details are provided in the Supplementary Appendix. The database was locked and data were unblinded after the final committee adjudication.

Secondary efficacy end points included the time to tuberculosis-associated IRIS; the duration of tuberculosis-associated IRIS; death (from any cause and attributed to tuberculosis-associated IRIS); hospitalization (for any cause and attributed to tuberculosis-associated IRIS); neurologic tuberculosis-associated IRIS; a composite end point of death, hospitalization, and hepatotoxicity; and adverse events resulting in a switch or interruption of antituberculosis treatment or ART. Secondary safety end points were severe infections and cancers, serious adverse events, adverse drug reactions, and grades 1 through 4 adverse events.

\section{STATISTICAL ANALYSIS}

Assuming that there would be a $35 \%$ incidence of tuberculosis-associated IRIS in the placebo group (approximating the 33\% incidence in the early ART group in the Cambodian Early versus Late Introduction of Antiretrovirals [CAMELIA] trial $^{15}$ ) and $50 \%$ fewer cases in the prednisone group than in the placebo group, we calculated that 110 patients would need to be enrolled in each group to provide $80 \%$ power to test for the difference between the groups in the incidence of tuberculosis-associated IRIS, at a two-sided significance level of $5 \%$. Assuming a $10 \%$ loss to follow-up, we planned to enroll a total of 240 patients.

The statistical analysis plan was finalized before the database was locked and unblinded. Analyses of the primary end point and the secondary efficacy end points used the intentionto-treat approach; safety analyses used the perprotocol approach. The analysis of the primary end point was performed with the use of the chisquare test. Secondary end points were analyzed with the use of chi-square, Fisher's exact, or Wilcoxon rank-sum tests, as appropriate. The duration of hospital stay was compared between the groups with the use of a zero-inflated Poisson model. In the comparison of the time from the initiation of ART to the development of tuberculosis-associated IRIS, death and loss to followup were considered to be competing risks, and a cause-specific hazard ratio was estimated, together with a $95 \%$ confidence interval. Cumulative incidence curves were plotted according to group. ${ }^{16}$ Subgroup analyses were prespecified. Adverse events were coded according to the Medical Dictionary for Regulatory Activities, version 19.1. All P values were two-sided, and P values of less than 0.05 were considered to indicate statistical significance. Analyses of secondary efficacy end points were not adjusted for multiple comparisons and therefore relative risks are reported with $95 \%$ confidence intervals, without $\mathrm{P}$ values. The $95 \%$ confidence intervals are not adjusted for multiple comparisons and may not be reproducible.

\section{RESULTS}

\section{TRIAL POPULATION}

From August 2013 through February 2016, a total of 321 adult patients were screened, and 240 were randomly assigned to receive prednisone (120 patients) or placebo (120 patients). The most frequent reasons for exclusion were hepatitis $B$ infection and previous receipt of ART (Fig. 1). One patient in each group did not receive the assigned regimen. Therefore, 240 patients constituted the intention-to-treat population, and 238 made up the per-protocol population. During 12 weeks of follow-up, 17 patients were lost to follow-up (although 8 of these patients returned to the trial clinic after 12 weeks), 1 withdrew, and 9 died. These outcomes occurred in similar numbers in the two groups.

The trial regimen was started on the same day that ART was started in 231 patients (4 patients started within 3 days before the start of ART, and 3 patients started 1 day after the start of ART). Of the 238 patients in the per-protocol population, $66(27.7 \%)$ stopped the trial regimen early; the most common reasons were prescription of open-label glucocorticoids to treat tuberculosis-associated IRIS (36 patients), diagnosis of severe infection (8 patients), discontinuation of the regimen at the time of hospital admission because the trial medication was left at home or owing to a misunderstanding (7 patients), and poor adherence to the trial regimen (5 patients) (Table S13 in the Supplementary Appendix). Dur- 


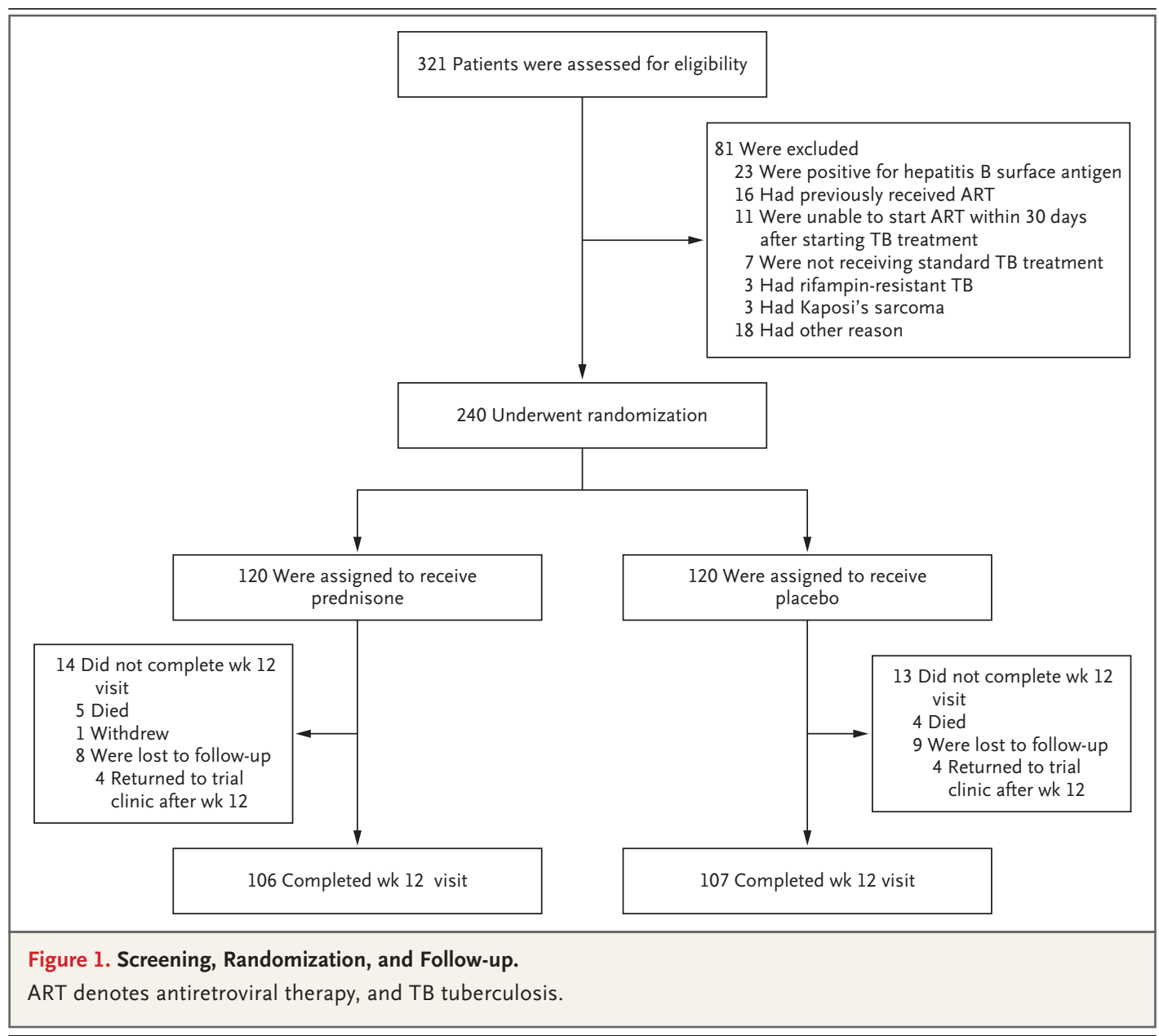

ing the trial, the ethics committee requested that the data be unblinded for 1 patient who died. Three patients ( 2 in the prednisone group and 1 in the placebo group) received a diagnosis of rifampin-resistant tuberculosis after trial entry and were switched to treatment for drug-resistant tuberculosis.

The baseline characteristics were balanced across the two groups (Table 1 ). The median age was 36 (interquartile range, 30 to 42 ), $60 \%$ were men, and $73 \%$ had microbiologically confirmed tuberculosis; the median CD4 count was 49 cells per microliter (interquartile range, 24 to 86), and the median HIV type 1 (HIV-1) RNA viral load was $5.5 \log _{10}$ copies per milliliter (interquartile range, 5.2 to 5.9). Patients had received antituberculosis treatment for a median of 17 days before starting ART. The ART regimen in 233 of the 240 patients $(97.1 \%)$ was tenofovir, emtricitabine (or lamivudine), and efavirenz.

\section{PRIMARY END POINT}

A total of 39 of 120 patients (32.5\%) in the prednisone group and 56 of 120 patients (46.7\%) in the placebo group received a diagnosis of paradoxical tuberculosis-associated IRIS (relative risk, $0.70 ; 95 \%$ confidence interval [CI], 0.51 to 0.96 ; $\mathrm{P}=0.03$ ) (Fig. 2A). The absolute difference between the groups in the incidence of tuberculosis-associated IRIS was 14.2 percentage points ( $95 \%$ CI, 1.9 to 26.4). The estimates of relative risk associated with prednisone in prespecified subgroups were similar to the overall estimate for the total trial population, but for most subgroups this difference was not significant (Table 2).

Among patients in the prednisone group who received a diagnosis of tuberculosis-associated IRIS, 10 had lymph-node involvement or abscess formation, 15 had new or worsening features on radiography, and 2 had new or worsening serositis. Fourteen patients met the INSHI criteria on 


\begin{tabular}{|c|c|c|}
\hline Characteristic & $\begin{array}{l}\text { Prednisone Group } \\
(\mathrm{N}=120)\end{array}$ & $\begin{array}{l}\text { Placebo Group } \\
\quad(N=120)\end{array}$ \\
\hline Median age (IQR) - yr & $36(31-42)$ & $36(29-42)$ \\
\hline Male sex - no. (\%) & $71(59.2)$ & $73(60.8)$ \\
\hline Median body-mass index (IQR) $\dagger$ & $21(19-24)$ & $21(19-24)$ \\
\hline Median CD4 count (IQR) - no. of cells $/ \mu \mathrm{l}$ & $51(27-84)$ & $49(23-88)$ \\
\hline Median HIV-I RNA viral load (IQR) - $\log _{10}$ copies $/ \mathrm{ml}$ & $5.5(5.2-5.9)$ & $5.6(5.2-5.9)$ \\
\hline Microbiologically confirmed TB - no. (\%) & $86(71.7)$ & $89(74.2)$ \\
\hline Median hemoglobin level $(\mathrm{IQR})-\mathrm{g} / \mathrm{dl}$ & $9.7(8.8-11.1)$ & $9.8(8.5-10.9)$ \\
\hline Median white-cell count (IQR) $-\times 10^{-9} /$ liter & $3.7(2.9-5.1)$ & $3.4(2.6-5.0)$ \\
\hline Median neutrophil count (IQR) $-\times 10^{-9} /$ liter & $2.3(1.5-3.1)$ & $2.0(1.4-2.9)$ \\
\hline Median platelet count (IQR) $-\times 10^{-9} /$ liter & $311(259-413)$ & $300(226-396)$ \\
\hline Median sodium level (IQR) - mmol/liter & $136(134-139)$ & $137(135-139)$ \\
\hline Median creatinine level (IQR) $-\mu \mathrm{mol} /$ liter & $57(50-66)$ & $59(50-70)$ \\
\hline Median total bilirubin level (IQR) $-\mu \mathrm{mol} /$ liter & $6(4-7)$ & $6(4-8)$ \\
\hline Median alanine aminotransferase level (IQR) - IU/liter & $26(18-38)$ & $28(20-40)$ \\
\hline Median alkaline phosphatase level (IQR) - IU/liter & $113(87-149)$ & $115(91-163)$ \\
\hline Median C-reactive protein level (IQR) - mg/liter & $10.9(4.0-30.1)$ & $10.7(4.6-29.9)$ \\
\hline Median Karnofsky performance score (IQR)』 & $90(80-90)$ & $90(80-90)$ \\
\hline $\begin{array}{l}\text { Median duration of TB treatment before initiation of ART } \\
(\text { (IQR) - days }\end{array}$ & $16(15-22)$ & $17(15-21)$ \\
\hline \multicolumn{3}{|c|}{$\begin{array}{l}\text { To convert the values for creatinine to milligrams per deciliter, divide by } 88.4 \text {. To convert the values for bilirubin to milli- } \\
\text { grams per deciliter, divide by } 17.1 \text {. ART denotes antiretroviral therapy, HIV-1 human immunodeficiency virus type } 1 \text {, } \\
\text { IQR interquartile range, and TB tuberculosis. } \\
\text { The body-mass index is the weight in kilograms divided by the square of the height in meters. } \\
\text { Microbiologically confirmed TB was defined as Mycobacterium tuberculosis detected on culture or with the use of the } \\
\text { Xpert MTB/RIF assay (Cepheid) or as positive acid-fast bacilli on smear microscopy. } \\
\text { The Karnofsky performance score ranges from } 0 \text { to } 100 \text {, with lower scores indicating greater disability. }\end{array}$} \\
\hline
\end{tabular}

the basis of two or three minor criteria alone. ${ }^{14}$ In the placebo group, 23 patients had lymph-node involvement or abscess formation, 26 had new or worsening features on radiography, 2 had new or worsening serositis, and 12 patients met the INSHI criteria on the basis of two or three minor criteria alone. No cases of neurologic tuberculosis-associated IRIS were diagnosed.

\section{SECONDARY EFFICACY END POINTS}

The cumulative incidence of tuberculosis-associated IRIS over 84 days was lower in the prednisone group than in the placebo group (hazard ratio, 0.61 ; $95 \%$ CI, 0.41 to 0.92 ) (Fig. 2B). Among the 95 patients who received a diagnosis of tuberculosis-associated IRIS, the median time to the onset of symptoms related to the syndrome was similar in the two groups (10 days [interquartile range, 5 to 13] in the prednisone group and 8 days [interquartile range, 4 to 12 ] in the placebo group).

Fewer patients in the prednisone group than in the placebo group met at least one major INSHI criterion (relative risk, 0.57 ; 95\% CI, 0.37 to 0.87 ) (Table 3). Fewer patients in the prednisone group than in the placebo group were treated for tuberculosis-associated IRIS with open-label glucocorticoids (relative risk, 0.47 ; 95\% CI, 0.27 to 0.81 ). When the analysis was restricted to the $95 \mathrm{pa}-$ tients who received a diagnosis of tuberculosisassociated IRIS, 16 of $39(41.0 \%)$ in the prednisone group and 34 of $56(60.7 \%)$ in the placebo group received treatment with open-label glucocorticoids (relative risk, $0.68 ; 95 \% \mathrm{CI}, 0.44$ to 1.04) (Table S10 in the Supplementary Appendix).

During the 12 weeks of follow-up, there were five deaths in the prednisone group and four in the placebo group $(\mathrm{P}=1.00)$; one death was attrib- 


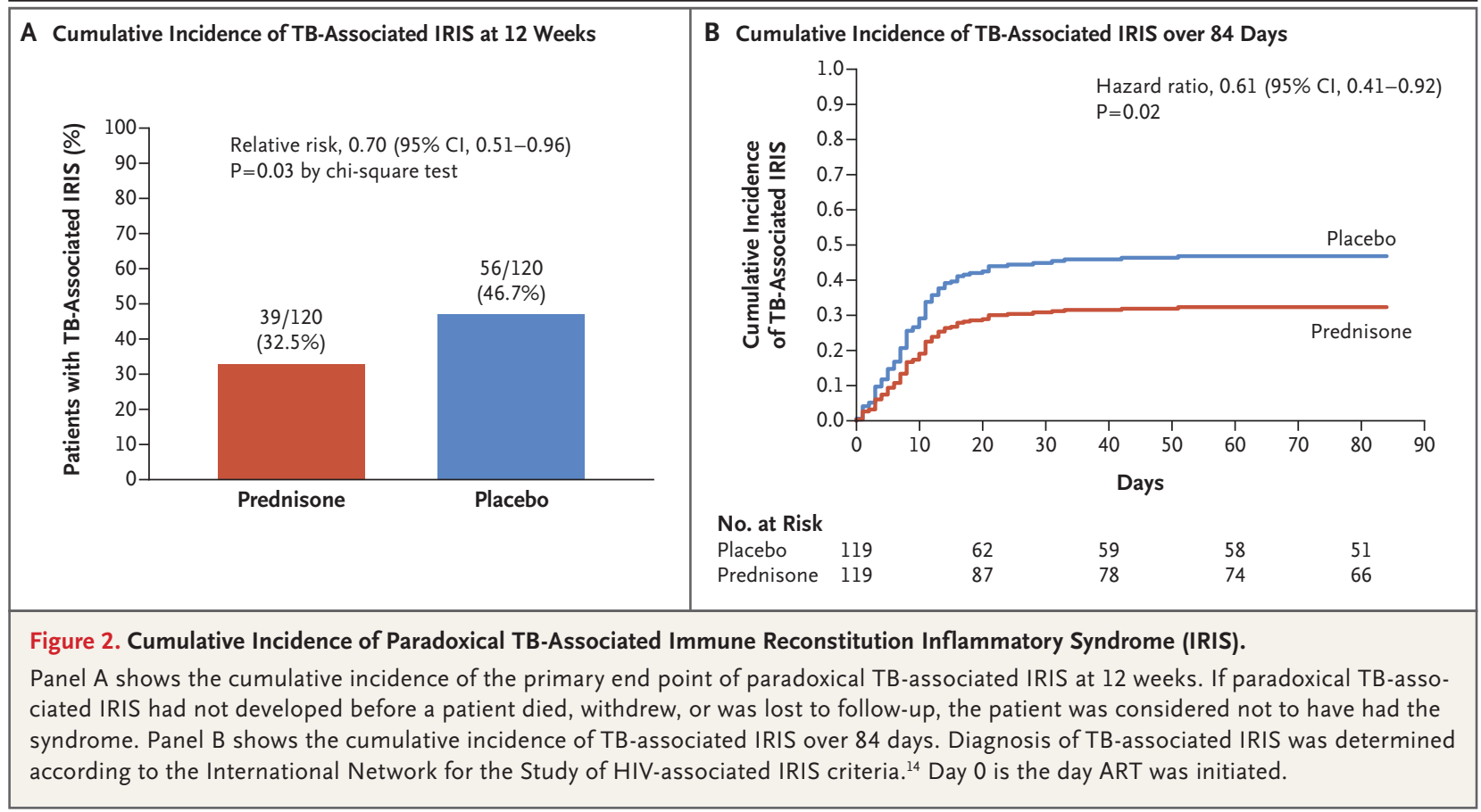

\begin{tabular}{|c|c|c|c|}
\hline \multirow[t]{2}{*}{ Subgroup } & $\begin{array}{l}\text { Prednisone Group } \\
\qquad(N=120)\end{array}$ & $\begin{array}{l}\text { Placebo Group } \\
\quad(N=120)\end{array}$ & $\begin{array}{c}\text { Relative Risk } \\
\text { (95\% CI) }\end{array}$ \\
\hline & \multicolumn{2}{|c|}{ no./total no. (\%) } & \\
\hline \multicolumn{4}{|l|}{ CD4 count at screening } \\
\hline$\leq 50$ cells $/ \mu \mathrm{l}$ & $28 / 60(46.7)$ & $37 / 62(59.7)$ & $0.78(0.56-1.10)$ \\
\hline$>50$ cells $/ \mu \mid$ & $11 / 60(18.3)$ & $19 / 58(32.8)$ & $0.56(0.29-1.07)$ \\
\hline \multicolumn{4}{|l|}{ HIV-1 RNA viral load at screening } \\
\hline$>100,000$ copies $/ \mathrm{ml}$ & $36 / 102(35.3)$ & $50 / 99(50.5)$ & $0.70(0.50-0.97)$ \\
\hline$\leq 100,000$ copies $/ \mathrm{ml}$ & $3 / 17(17.6)$ & $5 / 20(25.0)$ & $0.71(0.20-2.53)$ \\
\hline Microbiologically confirmed $\mathrm{TB} \uparrow$ & $33 / 86(38.4)$ & $43 / 89(48.3)$ & $0.79(0.56-1.12)$ \\
\hline $\begin{array}{l}\text { No rifampin-resistant TB diagnosed after } \\
\text { enrollment }\end{array}$ & $39 / 118(33.1)$ & $55 / 119(46.2)$ & $0.72(0.52-0.99)$ \\
\hline \multicolumn{4}{|c|}{$\begin{array}{l}\text { The primary end point was the development of paradoxical TB-associated IRIS within } 12 \text { weeks after enrollment accor } \\
\text { ing to the International Network for the Study of HIV-associated IRIS (INSHI) criteria. }{ }^{14} \\
\text { Microbiologically confirmed TB was defined as Mycobacterium tuberculosis detected on culture or with the use of the } \\
\text { Xpert MTB/RIF assay (Cepheid) or as positive acid-fast bacilli on smear microscopy. } \\
\text { In three patients (two in the prednisone group and one in the placebo group), rifampin-resistant TB was diagnosed af } \\
\text { ter enrollment, and these patients are excluded from the denominator in this analysis. }\end{array}$} \\
\hline
\end{tabular}

uted to tuberculosis-associated IRIS (Table S3 in the Supplementary Appendix). Fewer patients in the prednisone group than in the placebo group were hospitalized (17 vs. 27), but the difference was not significant. In a zero-inflated Poisson model, which included all patients, the duration of hospital stay was shorter in the prednisone group than in the placebo group. Fewer patients in the prednisone group than in the placebo group required a switch or interruption of ART or antituberculosis treatment owing to adverse events, but the difference was not significant; 37 events occurred among 29 patients (10 in the prednisone group and 19 in the placebo group). 
Table 3. Primary and Secondary End Points.*

\begin{tabular}{|c|c|c|c|c|}
\hline End Point & $\begin{array}{l}\text { Prednisone Group } \\
(\mathrm{N}=120)\end{array}$ & $\begin{array}{l}\text { Placebo Group } \\
(\mathrm{N}=120)\end{array}$ & $\begin{array}{l}\text { Relative Risk } \\
(95 \% \mathrm{CI})\end{array}$ & P Value \\
\hline \multicolumn{5}{|l|}{ Primary end point } \\
\hline TB-associated IRIS meeting INSHI criteria - no. (\%) & $39(32.5)$ & $56(46.7)$ & $0.70(0.51-0.96)$ & 0.03 \\
\hline \multicolumn{5}{|l|}{ Secondary efficacy end points } \\
\hline $\begin{array}{l}\text { TB-associated IRIS meeting at least } 1 \text { major INSHI } \\
\text { criterion - no. (\%) }\end{array}$ & $25(20.8)$ & $44(36.7)$ & $0.57(0.37-0.87)$ & \\
\hline Sustained TB-associated IRIS — no. (\%) $\dagger$ & $35(29.2)$ & $50(41.7)$ & $0.70(0.49-0.99)$ & \\
\hline Median duration of TB-associated IRIS (IQR) — days' & $49(31-97)$ & $35(19-82)$ & & \\
\hline $\begin{array}{l}\text { Open-label glucocorticoid treatment of TB-associated } \\
\text { IRIS - no. (\%) }\end{array}$ & $16(13.3)$ & $34(28.3)$ & $0.47(0.27-0.81)$ & \\
\hline Hospitalization for TB-associated IRIS — no. (\%) & $5(4.2)$ & $9(7.5)$ & $0.56(0.19-1.61)$ & \\
\hline Hospitalization for any cause - no. (\%) & $17(14.2)$ & $27(22.5)$ & $0.63(0.36-1.09)$ & \\
\hline Death from any cause - no. (\%) & $5(4.2)$ & $4(3.3)$ & $1.25(0.34-4.54)$ & 1.00 \\
\hline Death attributed to TB-associated IRIS — no. (\%) & 0 & $1(0.8)$ & $\begin{array}{l}\text { Could not be } \\
\text { calculated }\end{array}$ & 1.00 \\
\hline $\begin{array}{c}\text { Composite end point of death, hospitalization, and } \\
\text { hepatotoxicity — no. (\%) }\end{array}$ & $22(18.3)$ & $32(26.7)$ & $0.69(0.43-1.11)$ & \\
\hline $\begin{array}{l}\text { Interruption of ART, TB treatment, or both owing to } \\
\text { adverse event - no. (\%) }\end{array}$ & $10(8.3)$ & $19(15.8)$ & $0.53(0.26-1.08)$ & \\
\hline $\begin{array}{l}\text { Interruption of ART, TB treatment, or both owing to } \\
\text { drug-induced liver injury or rash - no. (\%) }\end{array}$ & $6(5.0)$ & $8(6.7)$ & $0.75(0.27-2.10)$ & \\
\hline \multicolumn{5}{|l|}{ Secondary safety end points } \\
\hline Severe infection - no./total no. (\%)』 & $11 / 119(9.2)$ & $18 / 119(15.1)$ & $0.61(0.30-1.24)$ & 0.23 \\
\hline Grade 3 clinical adverse event — no./total no. (\%) 9 & $33 / 119(27.7)$ & $53 / 119(44.5)$ & $0.62(0.44-0.89)$ & 0.01 \\
\hline Grade 4 clinical adverse event — no./total no. (\%) & $8 / 119(6.7)$ & $10 / 119(8.4)$ & $0.80(0.33-1.96)$ & 0.81 \\
\hline Serious adverse event - no./total no. (\%)\| & $24 / 119(20.2)$ & $30 / 119(25.2)$ & $0.80(0.50-1.28)$ & 0.44 \\
\hline Adverse drug reaction — no./total no.** & $22 / 119$ & $21 / 119$ & $1.05(0.61-1.80)$ & 1.00 \\
\hline Definitely related to trial regimen & $0 / 22$ & $0 / 21$ & & \\
\hline Probably related to trial regimen & $1 / 22$ & $2 / 21$ & & \\
\hline Possibly related to trial regimen & $21 / 22$ & $19 / 21$ & & \\
\hline \multicolumn{5}{|l|}{ CD4 count at week 12} \\
\hline No. of patients in analysis & 106 & 106 & & \\
\hline Median (IQR) - no. of cells $/ \mu \mathrm{l}$ & $164(97-226)$ & $150(100-226)$ & & 0.73 \\
\hline $\begin{array}{l}\text { Decrease in HIV-1 RNA viral load of }<2 \log _{10} \text { copies/ml } \\
\text { at week } 12-\text { no./total no. (\%) }\end{array}$ & $6 / 105(5.7)$ & $9 / 105(8.6)$ & $0.67(0.25-1.81)$ & 0.59 \\
\hline
\end{tabular}

* For contingency analyses, the chi-square test was used, unless the expected cell frequency was less than 6, in which case Fisher's exact test was used. The Wilcoxon rank-sum test was used to compare continuous variables.

$\dagger$ Sustained TB-associated IRIS was defined as a duration of more than 14 days, the need for open-label glucocorticoid treatment, or both. For the duration of TB-associated IRIS, we excluded three participants, all of whom were receiving glucocorticoid treatment: two participants with ongoing TB-associated IRIS who died and one participant with ongoing TB-associated IRIS who was lost to follow-up.

$\mp$ Additional information on secondary safety end points is provided in Tables S1, S5 through S8, and S16 through S19 in the Supplementary Appendix.

$\int$ Severe infections were considered to be acquired immunodeficiency syndrome (AIDS)-defining illnesses or invasive bacterial infections; 33 severe infections occurred among 29 participants: cryptococcal meningitis in 3 ( 1 in the prednisone group and 2 in the placebo group), esophageal candidiasis in 10 (4 in the prednisone group and 6 in the placebo group), sepsis in 7 (4 in the prednisone group and 3 in the placebo group), pneumonia in 7 ( 2 in the prednisone group and 5 in the placebo group), pyelonephritis in 1 patient in the placebo group, Clostridium difficile colitis in 3 patients in the placebo group, and dysentery in 2 ( 1 in the prednisone group and 1 in the placebo group).

q Adverse events of grade 1, 2, 3, and 4 were defined according to the Division of AIDS Table for Grading the Severity of Adult and Pediatric Adverse Events, version 1.0. In the prednisone group, there were 517 clinical events of grade 1 severity, 167 events of grade 2 severity, 66 events of grade 3 severity, and 10 events of grade 4 severity. In the placebo group, there were 660 clinical events of grade 1 severity, 218 events of grade 2 severity, 112 events of grade 3 severity, and 12 events of grade 4 severity.

|| Serious adverse events were defined according to International Conference on Harmonisation guidelines for Good Clinical Practice definitions. In the prednisone group, there were 55 serious adverse events among 24 patients; in the placebo group, there were 56 serious adverse events among 30 patients.

** Adverse drug reactions were defined as adverse events determined by the trial doctor to be possibly, probably, or definitely related to the trial regimen but not to open-label prednisone. A total of 45 probable or possible adverse drug reactions were reported among 43 patients. None of these were categorized as serious adverse events. 


\section{SECONDARY SAFETY END POINTS}

Fewer patients in the prednisone group than in the placebo group had clinical adverse events of grade 3 , but there was no significant difference in grade 4 events. There were also no significant differences between the groups in grade 3 or 4 laboratory adverse events. A total of 33 severe infections (defined as new acquired immunodeficiency syndrome [AIDS]-defining illnesses or invasive bacterial infections) were diagnosed in 29 patients: $11(9.2 \%)$ in the prednisone group and $18(15.1 \%)$ in the placebo group $(\mathrm{P}=0.23)$. There was no significant between-group difference in prespecified adverse events related to glucocorticoid treatment (18 events in the prednisone group and 23 in the placebo group). There was no significant difference between the groups in CD4 cell count or HIV-1 RNA suppression at week 12.

Vital status at 1 year was available for 239 patients: 18 had died ( 8 in the prednisone group and 10 in the placebo group). Status with respect to cancer was ascertained for 220 patients at 1 year. One case of Kaposi's sarcoma was diagnosed: the patient (who was in the placebo group) had received open-label prednisone starting at week 2 to treat presumed tuberculosis-associated IRIS and then discontinued ART at week 20; the patient returned to the trial clinic at week 28 with a Kaposi's sarcoma lesion that resolved after reinitiation of ART. Additional information on secondary efficacy end points, adverse events, and other safety variables is provided in the Supplementary Appendix.

\section{DISCUSSION}

Prophylactic prednisone during the first 4 weeks after the initiation of ART in adult patients at high risk for tuberculosis-associated IRIS resulted in a $30 \%$ lower incidence of tuberculosis-associated IRIS than placebo. Prednisone use was not associated with an excess risk of severe infections, cancers, or adverse events. Prednisone might exert its effect by suppressing the symptoms of only mild cases of tuberculosis-associated IRIS, but our findings suggest that this was not the case. We found that prescription of openlabel glucocorticoids, which are generally prescribed to treat patients with more severe tuberculosis-associated IRIS, was 53\% less in the prednisone group than in the placebo group, and a significantly smaller proportion of patients in the prednisone group than in the placebo group met at least one major INSHI criterion.

The development of tuberculosis-associated IRIS in $47 \%$ of the patients in the placebo group in our trial is higher than the pooled estimate of $18 \%$ from a meta-analysis of 40 cohort studies ${ }^{4}$; our finding reflects our enrollment of patients at high risk for tuberculosis-associated IRIS who had low CD4 counts and short intervals between starting antituberculosis treatment and starting ART. A study in India involving patients with a similar high-risk profile showed an incidence of tuberculosis-associated IRIS of 54\%. ${ }^{17}$ In one previous trial of a prophylactic strategy for IRIS, the CCR 5 antagonist maraviroc was evaluated for prevention of all causes of IRIS, but the trial did not show a lower incidence of IRIS in the group that received maraviroc than in the group that received placebo. ${ }^{18}$

The dose of prednisone used in our trial was relatively low, considering that rifampin reduces plasma exposure to prednisone (clearance is increased $45 \%$ by rifampin). ${ }^{19}$ In several rheumatologic conditions (e.g., rheumatoid arthritis), lowdose glucocorticoids are effective, ${ }^{20-22}$ which indicates that there is a substantial antiinflammatory effect. Lower doses of glucocorticoids may cause less harm than higher doses, which is an important consideration in the prevention of tuberculosis-associated IRIS.

Kaposi's sarcoma was diagnosed in one patient in the placebo group who received openlabel prednisone and in whom ART was interrupted. By contrast, in the Investigation of the Management of Pericarditis (IMPI) trial, prednisolone for the treatment of tuberculous pericarditis resulted in a significant excess risk of HIVrelated cancers. ${ }^{9}$ The differences between our trial and the IMPI trial were that we used a lower dose of prednisone for a shorter duration, and all the patients in our trial started ART at the same time that they started the trial regimen. In the IMPI trial, $22 \%$ of HIV-positive patients were receiving ART at entry, and $72 \%$ by 6 months. ${ }^{9}$ Our findings show that short-course glucocorticoids can be used safely in patients receiving ART.

We found a trend toward fewer interruptions of ART or antituberculosis treatment in the prednisone group than in the placebo group. This finding is consistent with two previous observations from placebo-controlled trials of adjunctive glucocorticoids: in one trial, there were fewer events of drug-induced liver injury in the group 
assigned to dexamethasone for the treatment of tuberculous meningitis than in the group assigned to placebo ${ }^{23}$; in another trial, in which methylprednisolone was assessed for the treatment of AIDS-related pneumocystis pneumonia, fewer participants in the methylprednisolone group than in the placebo group stopped trimethoprim-sulfamethoxazole owing to hypersensitivity. ${ }^{24}$ Reducing the risk of adverse drug reactions, which are more frequent in HIV-infected patients, ${ }^{25}$ is another potential benefit of glucocorticoids. The lower incidence of grade 3 clinical adverse events in the prednisone group than in the placebo group may reflect that these patients had fewer symptoms related to tuberculosisassociated IRIS and fewer adverse drug reactions to ART and antituberculosis treatment.

Our trial has several limitations. First, our findings may not be generalizable to inpatients; however, most patients with HIV-associated tuberculosis are ambulatory. Our trial site was a busy government-run clinic in which guidelines for HIV and tuberculosis management are aligned with WHO guidelines. Second, our trial was not sufficiently powered to detect mortality differences. Third, a quarter of our patients had clinically diagnosed tuberculosis, and they may have had other opportunistic diseases. However, this is unlikely, since we enrolled only patients with a clinical diagnosis of tuberculosis who had a response to antituberculosis treatment. Fourth, the $30 \%$ lower incidence of tuberculosis-associated IRIS in the prednisone group than in the placebo group was smaller than the $50 \%$ predicted in our sample-size calculation, but the difference was statistically significant because the event rate was higher than predicted.

In conclusion, we found that among adult HIV-infected patients receiving antituberculosis treatment who were at high risk for tuberculosisassociated IRIS, the incidence of tuberculosis- associated IRIS was lower among those who received a 4-week course of prednisone, prescribed when ART was started, than among those who received placebo. Future studies could evaluate higher doses of prednisone or target prednisone prophylaxis in patients identified as having a high risk of tuberculosis-associated IRIS on the basis of immune biomarkers. ${ }^{17}$

The opinions, findings, and conclusions expressed are those of the authors alone and are not necessarily those of the funders.

Supported by the European and Developing Countries Clinical Trials Partnership through a Strategic Primer Grant (SP.2011.41304.074) that was awarded to the University of Cape Town, the Institute of Tropical Medicine, and Imperial College London, funding from the Department of Science and Technology of the government of South Africa, grants (098316, 084323, 104803, 203135) from the Wellcome Trust, and a doctoral fellowship (awarded to Dr. Stek) from the Institute for Tropical Medicine. Dr. Meintjes is supported by a grant (64787) from the South African Research Chairs Initiative of the Department of Science and Technology and the National Research Foundation (NRF) of South Africa, NRF incentive funding (UID: 85858), and the South African Medical Research Council through its TB and HIV Collaborating Centres Programme, with a grant (RFA\# SAMRC-RFA-CC: TB/HIV/AIDS-01-2014) funded by the National Department of Health. Dr. Wilkinson is supported by a grant (10218) from the Francis Crick Institute, which is funded by Wellcome, Research Councils UK, and Cancer Research UK.

No potential conflict of interest relevant to this article was reported.

Disclosure forms provided by the authors are available with the full text of this article at NEJM.org.

We thank the clinical and management staff of the Western Cape Government Department of Health and the City of Cape Town for their support of the trial; the trial team (Alison Swartz, Edwin Wouters, Christiana Nöstlinger, Yolisa Sigila, Monica Magwayi, Nobom Masimini, Nashreen Omar-Davies, Loraine Swanepoel, Rene Goliath, Kathryn Wood, Holly Gathercole, Nomvula Makade, and Antoneta Mashinyira); Joris Menten for statistical advice during the planning phase; Danielle Van Melle (ITM) for initial administrative support to the trial management group; the clinic staff (Shaheed Matthee, Jan Kuene, and all medical, nursing, and counseling staff); Bill Burman (independent member of the trial steering committee); Colleen Whitelaw (independent pharmacist); Tom Boyles (independent member of the end-points committee); Gavin Churchyard (chair of the data and safety monitoring board); Julian Elliott and Guy Thwaites (members of the data and safety monitoring board); Maia Lesosky (independent statistician for the data and safety monitoring board); Michael Hubbard for database development and maintenance; and Andrew Boulle for assistance with data acquisition.

\section{APPENDIX}

The authors' full names and academic degrees are as follows: Graeme Meintjes, M.B., Ch.B., Ph.D., Cari Stek, M.D., Lisette Blumenthal, M.B., Ch.B., Friedrich Thienemann, M.D., Charlotte Schutz, M.B., Ch.B., Jozefien Buyze, Ph.D., Raffaella Ravinetto, Pharm.D., Ph.D., Harry van Loen, M.Sc., Amy Nair, M.Sc., Amanda Jackson, B.Sc., Robert Colebunders, M.D., Ph.D., Gary Maartens, M.B., Ch.B., Robert J. Wilkinson, F.Med.Sci., and Lutgarde Lynen, M.D., Ph.D.

The authors' affiliations are as follows: the Wellcome Centre for Infectious Diseases Research in Africa, Institute of Infectious Disease and Molecular Medicine (G. Meintjes, C. Stek, L.B., F.T., C. Schutz, A.N., A.J., R.J.W.), the Department of Medicine (G. Meintjes, C. Stek, F.T., C. Schutz, R.J.W.), and the Division of Clinical Pharmacology, Department of Medicine (G. Maartens), University of Cape Town, Cape Town, South Africa; the Department of Clinical Sciences, Institute of Tropical Medicine, Antwerp, Belgium (C. Stek, J.B., R.R., H.L., R.C., L.L.); the Department of Internal Medicine, University Hospital of Zurich, Zurich, Switzerland (F.T.); and the Department of Medicine, Imperial College London and the Francis Crick Institute, London (R.J.W.). 


\section{REFERENCES}

1. Avila D, Althoff KN, Mugglin C, et al. Immunodeficiency at the start of combination antiretroviral therapy in lowmiddle-, and high-income countries. J Acquir Immune Defic Syndr 2014;65(1):e8-e16. 2. Ford N, Shubber Z, Meintjes G, et al. Causes of hospital admission among people living with HIV worldwide: a systematic review and meta-analysis. Lancet HIV 2015;2(10):e438-e444.

3. Lai RP, Meintjes G, Wilkinson RJ. HIV-1 tuberculosis-associated immune reconstitution inflammatory syndrome. Semin Immunopathol 2016;38:185-98.

4. Namale PE, Abdullahi LH, Fine S, Kamkuemah M, Wilkinson RJ, Meintjes G. Paradoxical TB-IRIS in HIV-infected adults: a systematic review and meta-analysis. Future Microbiol 2015;10:1077-99.

5. Uthman OA, Okwundu C, Gbenga K, et al. Optimal timing of antiretroviral therapy initiation for HIV-infected adults with newly diagnosed pulmonary tuberculosis: a systematic review and metaanalysis. Ann Intern Med 2015;163:32-9.

6. Consolidated guidelines on the use of antiretroviral drugs for treating and preventing HIV infection: recommendations for a public health approach. 2nd ed. Geneva: World Health Organization, 2016.

7. Dooley DP, Carpenter JL, Rademacher S. Adjunctive corticosteroid therapy for tuberculosis: a critical reappraisal of the literature. Clin Infect Dis 1997;25:872-87.

8. Critchley JA, Young F, Orton L, Garner P. Corticosteroids for prevention of mortality in people with tuberculosis: a systematic review and meta-analysis. Lancet Infect Dis 2013;13:223-37.

9. Mayosi BM, Ntsekhe M, Bosch J, et al. Prednisolone and Mycobacterium indicus pranii in tuberculous pericarditis. $\mathrm{N}$ Engl J Med 2014;371:1121-30.

10. Elliott AM, Luzze H, Quigley MA, et al.
A randomized, double-blind, placebo-controlled trial of the use of prednisolone as an adjunct to treatment in HIV-1-associated pleural tuberculosis. J Infect Dis 2004; 190:869-78.

11. Meintjes G, Wilkinson RJ, Morroni C, et al. Randomized placebo-controlled trial of prednisone for paradoxical tuberculosisassociated immune reconstitution inflammatory syndrome. AIDS 2010;24:2381-90. 12. Stek C, Schutz C, Blumenthal L, et al. Preventing paradoxical tuberculosis-associated immune reconstitution inflammatory syndrome in high-risk patients: protocol of a randomized placebo-controlled trial of prednisone (PredART Trial). JMIR Res Protoc 2016;5(3):e173.

13. Lawn SD, Myer L, Bekker LG, Wood R. Tuberculosis-associated immune reconstitution disease: incidence, risk factors and impact in an antiretroviral treatment service in South Africa. AIDS 2007;21:335-41. 14. Meintjes G, Lawn SD, Scano F, et al. Tuberculosis-associated immune reconstitution inflammatory syndrome: case definitions for use in resource-limited settings. Lancet Infect Dis 2008;8:516-23.

15. Blanc FX, Sok T, Laureillard D, et al. Earlier versus later start of antiretroviral therapy in HIV-infected adults with tuberculosis. N Engl J Med 2011;365:1471-81.

16. Fine JP, Gray RJ. A proportional hazards model for the subdistribution of a competing risk. J Am Stat Assoc 1999;94: 496-509.

17. Narendran G, Andrade BB, Porter BO, et al. Paradoxical tuberculosis immune reconstitution inflammatory syndrome (TB-IRIS) in HIV patients with culture confirmed pulmonary tuberculosis in India and the potential role of IL- 6 in prediction. PLoS One 2013;8(5):e63541.

18. Sierra-Madero JG, Ellenberg SS, Rassool MS, et al. Effect of the CCR 5 antagonist maraviroc on the occurrence of immune reconstitution inflammatory syndrome in HIV (CADIRIS): a double-blind, randomised, placebo-controlled trial. Lancet HIV 2014;1(2):e60-e67.

19. McAllister WA, Thompson PJ, Al-Habet SM, Rogers HJ. Rifampicin reduces effectiveness and bioavailability of prednisolone. Br Med J (Clin Res Ed) 1983;286: 923-5.

20. Bootsma H, Spronk P, Derksen R, et al. Prevention of relapses in systemic lupus erythematosus. Lancet 1995;345:1595-9.

21. Pincus T, Cutolo M. Clinical trials documenting the efficacy of low-dose glucocorticoids in rheumatoid arthritis. Neuroimmunomodulation 2015;22:46-50.

22. Tseng CE, Buyon JP, Kim M, et al. The effect of moderate-dose corticosteroids in preventing severe flares in patients with serologically active, but clinically stable, systemic lupus erythematosus: findings of a prospective, randomized, doubleblind, placebo-controlled trial. Arthritis Rheum 2006;54:3623-32.

23. Thwaites GE, Nguyen DB, Nguyen HD, et al. Dexamethasone for the treatment of tuberculous meningitis in adolescents and adults. N Engl J Med 2004;351:1741-51.

24. Walmsley S, Levinton C, Brunton J, et al. A multicenter randomized doubleblind placebo-controlled trial of adjunctive corticosteroids in the treatment of Pneumocystis carinii pneumonia complicating the acquired immune deficiency syndrome. J Acquir Immune Defic Syndr Hum Retrovirol 1995;8:348-57.

25. Yee D, Valiquette C, Pelletier M, Parisien I, Rocher I, Menzies D. Incidence of serious side effects from first-line antituberculosis drugs among patients treated for active tuberculosis. Am J Respir Crit Care Med 2003;167:1472-7.

Copyright @ 2018 Massachusetts Medical Society. 